\title{
Visualization and analysis of lipopolysaccharide distribution in binary phospholipid bilayers
}

\author{
María Florencia Henning a , Susana Sanchez ${ }^{\mathrm{b}}$, Laura Bakás ${ }^{\mathrm{a}, \mathrm{c}, * *}$ \\ a Instituto de Investigaciones Bioquímicas La Plata (INIBIOLP), CCT-La Plata, CONICET, Facultad de Ciencias Médicas, UNLP, Calles 60 y 120,1900 La Plata, Argentina \\ ${ }^{\mathrm{b}}$ Laboratory for Fluorescence Dynamics, University of California-Irvine, Irvine, CA, USA \\ ${ }^{\mathrm{c}}$ Departamento de Ciencias Biológicas, Facultad de Ciencias Exactas, UNLP, Calles 47 y 115, 1900 La Plata, Argentina
}

\section{A R T I C L E I N F O}

Article history:

Received 17 March 2009

Available online 24 March 2009

\section{Keywords:}

Membrane raft

Giant unilamellar vesicles

Two-photon microscopy

LAURDAN

FITC-LPS

Detergent solubilization

Triton X-100

LAURDAN generalized polarization

Cholesterol

Phase segregation

\begin{abstract}
A B S T R A C T
Lipopolysaccharide (LPS) is an endotoxin released from the outer membrane of Gram-negative bacteria during infections. It have been reported that LPS may play a role in the outer membrane of bacteria similar to that of cholesterol in eukaryotic plasma membranes.

In this article we compare the effect of introducing LPS or cholesterol in liposomes made of dipalmitoylphosphatidylcholine/dioleoylphosphatidylcholine on the solubilization process by Triton X-100. The results show that liposomes containing LPS or cholesterol are more resistant to solubilization by Triton X-100 than the binary phospholipid mixtures at $4{ }^{\circ} \mathrm{C}$.

The LPS distribution was analyzed on GUVs of DPPC:DOPC using FITC-LPS. Solid and liquid-crystalline domains were visualized labeling the GUVs with LAURDAN and GP images were acquired using a twophoton microscope. The images show a selective distribution of LPS in gel domains.

Our results support the hypothesis that LPS could aggregate and concentrate selectively in biological membranes providing a mechanism to bring together several components of the LPS-sensing machinery.
\end{abstract}

(c) 2009 Elsevier Inc. All rights reserved.

\section{Introduction}

Lipopolysaccharide represents a major virulence factor of Gram-negative bacteria, which can cause septic shock in mammals including man.

The LPS released during division and/or death of bacteria is ingested by macrophages, which act as an early defense against bacterial pathogens by phagocytosing and degrading microorganisms [1].

It was previously demonstrated that Brucella abortus LPS captured by macrophages is recycled back to the plasma membrane where it was found associated in large clusters named macrodomains [2], which were characterized by microscopy and biochemistry approaches demonstrating that LPS domains segregate several lipid-raft components, LPS-binding proteins and MHC-II molecules. Forestier et al. [3] suggested that $B$. abortus LPS is responsible for the formation of rigid surface

\footnotetext{
* Corresponding author. Address: Instituto de Investigaciones Bioquímicas La Plata (INIBIOLP), CCT-La Plata, CONICET, Facultad de Ciencias Médicas, UNLP, Calles 60 y 120, 1900 La Plata, Argentina. Fax: +54 2214258988.

E-mail address: lbakas@biol.unlp.edu.ar (L. Bakás).
}

membrane complexes and also for the reorganization of the cell membrane to prevent that MHC-II proteins reach functional lipid rafts, by sequestering these molecules in dense nonfunctional structures. The domain segregation of several lipid-raft components and the formation of rigid surface membrane complexes suggest a role for LPS, in bacterial membrane, similar to that of cholesterol in plasma membrane of eukaryotic cells.

A number of more recent reports indicate that Uropathogenic Escherichia coli can act as an opportunistic intracellular pathogen [4]. In spite of the capabilities of $E$. coli to internalize into host cells, nothing is reported about how is processed the LPS inside the cell and if it is recycle back to the membrane.

In this report the capability of $E$. coli LPS to segregate into particular domains is explored. We used giant unilamellar vesicles (GUVs) made of the ternary mixtures DOPC:DPPC:Chol and DOPC:DPPC:LPS both in a 1:1:1 molar ratio and as control the binary mixture DOPC:DPPC.

Our results show that LPS incorporated into membranes impair resistance to detergent solubilization as well as cholesterol. Using fluorescein labeled LPS we show that LPS preferentially segregate in more ordered domains as well as cholesterol. 


\section{Materials and methods}

\section{Methods}

Preparation of multilamellar vesicles (MLVs). Pure lipids were dissolved in chloroform and mixed at the desired molar ratio. Solvent was removed by evaporation at room temperature under nitrogen stream and dried lipids were then hydrated at room temperature in $10 \mathrm{mM}$ Tris, $150 \mathrm{mM} \mathrm{NaCl}, 0.01 \%$ EDTA, pH 8 buffer (buffer A) obtaining MLVs.

MLVs of DPPC:DOPC:LPS (1:1:1 molar ratio) were made using the protocol published by Dijkstra et al. [5]. Briefly, the lipid mixture (DOPC:DPPC 1:1 molar ratio) in chloroform was dried under nitrogen stream, next the lipids were suspended in $2 \mathrm{ml}$ of water containing the desired amount of LPS $(1.73 \mathrm{mg})$. After vortexing and heating at $45^{\circ} \mathrm{C}$, the lipid suspension was sonicated for two $15 \mathrm{~s}$ periods. The resulting solution was dried and suspended in $1 \mathrm{ml}$ of buffer A. The final concentration of total lipids was $2 \mathrm{mg} /$ $\mathrm{ml}$.

Preparation of giant unilamellar vesicles (GUVs). GUVs were prepared using a modified version [6] of the protocol developed by Angelova and Dimitrov [7]. Phospholipid stock solutions were prepared in chloroform at concentration of $0.2 \mathrm{mg} / \mathrm{ml}$. Three mixtures were used: DPPC:DOPC (1:1 molar ratio), DPPC:DOPC:FITC-LPS (1:1:1 molar ratio) and DPPC:DOPC:Chol (1:1:1).

LAURDAN was added to the sample chamber after the vesicles were formed to a final phospholipid/LAURDAN molar ratio of 100:1.

Turbidity kinetics measurements. MLVs suspensions made of DPPC:DOPC (1:1 molar ratio), DPPC:DOPC:LPS (1:1:1 molar ratio) or DPPC:DOPC:Chol (1:1:1 molar ratio) were left to equilibrate for $3 \mathrm{~h}$ at $4{ }^{\circ} \mathrm{C}$ after Triton X-100 was added at 3:1 (detergent:lipids molar ratio) in the same buffer solution. MLVs concentration was $0.2 \mathrm{mg} / \mathrm{ml}$ in lipid.

The solubilization process was followed on time by the decrease of turbidity in a spectrofluorometer (SLM-aminco 4800) at $500 \mathrm{~nm}$ for excitation and emission wavelength, with continuous sample stirring and the temperature kept at $4{ }^{\circ} \mathrm{C}$. Turbidity values were normalized by setting $100 \%$ as the turbidity of MLV suspensions and $0 \%$ correspond to buffer.

LAURDAN generalized polarization (GP). LAURDAN is used as a membrane probe because of its large excited-state dipole moment, which results in its ability to report the extent of water penetration into the bilayer surface as a result of the dipolar relaxation effect [8]. Water penetration has been correlated with lipid packing and membrane fluidity [9]. The emission spectrum of LAURDAN in a single phospholipid bilayer is centered at $440 \mathrm{~nm}$ when the membrane is in the gel phase and at 490 when it is in the liquidcrystalline phase. The GP function quantified this emission shift and is given by:

$\mathrm{GP}=I_{440}-I_{490} / I_{440}+I_{490}$

where $I_{440}$ and $I_{490}$ are the emission intensities at 440 and $490 \mathrm{~nm}$, respectively. A full discussion of the use and mathematical significance of GP can be found in the literature [9]. If LAURDAN is solubilized in a lipid structure, such as a liposome, its spectrum will move according to the water content of the bilayer.

LAURDAN generalized polarization (GP) images. To obtain a GP image, an excitation wavelength of $780 \mathrm{~nm}$ and a two-channel detection system with the corresponding filters on the emission were used. A two-photon excitation is needed due to the extensive photobleaching of the dye with one-photon excitation [10].

Two simultaneous $256 \times 256$ pixel images are obtained from the vesicle (image channel 1 and image channel 2), one for each region of the LAURDAN spectra [10-13]. This two intensity images are processed applying the GP formula (Eq. (1)) to each pixel of the image. The resulting image (GP image) can be represented in a histogram of the number of pixels with a given GP.

Instrumentation. We used a two-photon excitation microscope designed at the Laboratory for Fluorescence Dynamics [14,15]. In this system the two-photon excitation source is a titanium-sapphire laser (Mira 900; Coherent, Palo Alto, CA) pumped by a frequency-doubled Nd:Vanadate laser (Verdi; Coherent, Palo Alto, CA) set at $780 \mathrm{~nm}$.

The fluorescence emission was observed through a 350-600 nm bandpass filter from (BG39 filter; Chroma Technology, Brattleboro, VT). A commercial version (ISS card) of a card designed at the Laboratory for Fluorescence Dynamics [13] was used to acquire the counts. A two-channel detection system was used for the LAURDAN GP images. The fluorescence was split into red and blue channels using a Chroma Technology 470DCXR-BS dichroic beam splitter in the emission path. Interference filters (Ealing 490 and Ealing 440) were placed in the emission paths to further isolate the red and blue parts of the emission spectrum. Separate detectors were used for each channel to simultaneously collect 490 and $440 \mathrm{~nm}$ emission. Red and blue images were collected simultaneously and then recombined to form the GP image of the sample using the SimFCS program (Laboratory for Fluorescence Dynamics, Irvine, CA).

Intensity images. Fluorescein labeled LPS (FITC-LPS) was used to study the distribution of the molecule in the liposome at different temperatures. After the GUVs were grown with the labeled LPS, intensity images were taken using the same instrumental set up described previously, exciting at $780 \mathrm{~nm}$ and using the channel with the filter centered at $490 \mathrm{~nm}$ to collect the emission. To obtain a GP image of the same GUV, LAURDAN was added into the solution.

\section{Results and discussion}

The main focus of this report is to compare the properties of LPS and cholesterol when they are incorporated into the same type of lipid bilayer.

\section{Solubilization by Triton $\mathrm{X}-100$}

The first experiments were designed to compare the solubilization kinetics of MLVs made of DOPC:DPPC (1:1) and DOPC:DPPC containing LPS or cholesterol in a 1:1:1 molar ratio by Triton X100 . The key parameter controlling the degree of solubilization is the ratio between the concentrations of detergent to lipid, however solubilization also depend strongly upon the physical state of the lipid [16].

The decrease in light scattering after addition of Triton X-100 at 3:1 (detergent:lipids) molar ratio at $4{ }^{\circ} \mathrm{C}$ was followed in time and it is shown in Fig. 1. Turbidity decreased about $10 \%$ in the ternary mixture containing LPS or cholesterol and 60\% on MLVs composed by the binary phospholipid mixtures. The experiments were also done at $37^{\circ} \mathrm{C}$ and the protective effect of both (LPS and cholesterol) in comparison with the binary mixture was also observed (data not shown).

The solubilization experiments clearly show that both LPS and cholesterol confer to the MLVs a resistance to solubilization by Triton X-100. A three-stage model has been proposed to describe lipid detergent interaction during solubilization [16].

The resistance to detergent solubilization is related to the molecular packing of lipid molecules [17]: bilayers in the liquid order state showed to be less susceptible to solubilization than those in the liquid-disordered or fluid state [18]. The resistance to solubilization by TX-100 when cholesterol is present in the bilayer is 


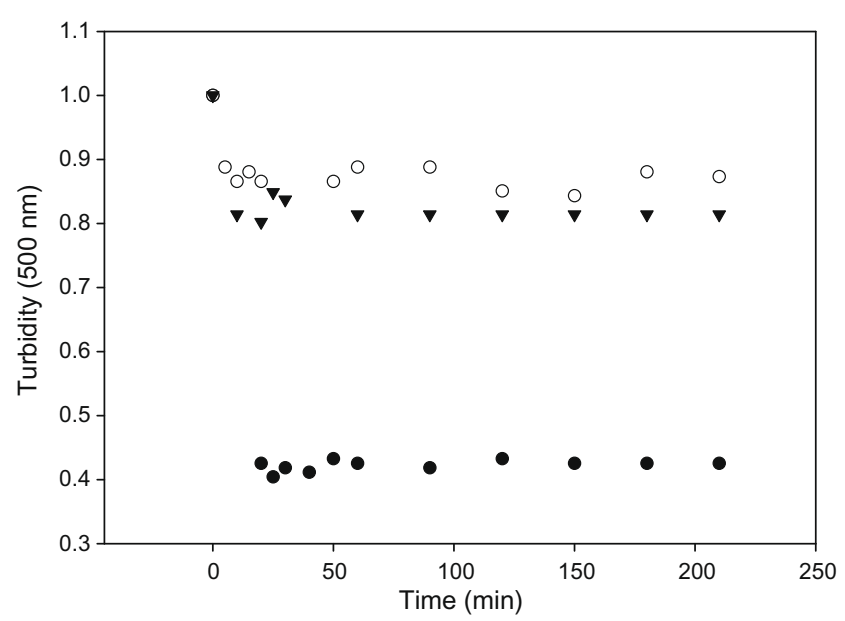

Fig. 1. Effect of LPS on lipid vesicles solubilization by Triton X-100. MLVs composed of DOPC:DPPC (1:1 molar ratio) ( $\bullet$ ), DOPC:DPPC:LPS (1:1:1 molar ratio) ( $\mathbf{\nabla})$, DOPC:DPPC:Chol (1:1:1 molar ratio) $(\bigcirc)$ in buffer A were treated with Triton X-100 at 3:1 (detergent:lipids molar ratio) and the turbidity decrease was measured by light scattering in an spectrofluorometer with excitation and emission wavelength of $500 \mathrm{~nm}$, at $4{ }^{\circ} \mathrm{C}$ for $3 \mathrm{~h}$. The intensity at time 0 was normalized to $1 \mathrm{U}$ in all samples.

related to the structure and mechanical properties of the bilayer. The introduction of cholesterol reduces the area per phospholipid molecules and also increases the isothermal area compressibility modulus (Ka) [19]. As Cholesterol, LPS decrease the area per phospholipid molecule or area per hydrocarbon chain $\left(A_{\mathrm{lc}}\right)$. The area per hydrocarbon chain has been measured to be $26 \AA^{2}$ for LPS which can be compared to an $A_{\mathrm{lc}}$ of $\approx 26 \AA^{2}$ for a fully hydrated $1: 1 \mathrm{EPC} /$ cholesterol mixture [20] but it is small compared to hydrocarbon chain areas determined for phospholipids with unsaturated chains typical of phospholipids found in biological membranes, which vary between 32 and $41 \AA^{2}[21,22]$.

Other possible explanation to similar detergent resistance of bilayer containing LPS and Cholesterol may be founded in the wellknown dependence between the formed structures and the shape of the lipids and detergents [23]. It is known that micelles formation requires molecules with a cone shape in which the polar head group occupies a space with a larger cross section than that of the hydrocarbon. Cholesterol and lipid A (known as the region of LPS molecule inserted into the membrane) have inverted cone shape [24] in which the hydrocarbon chains occupies a space with a larger cross section than that of polar head groups, therefore they do

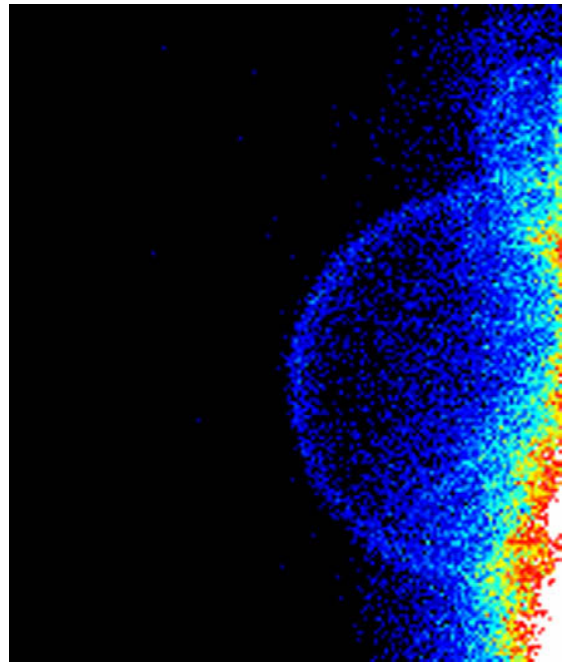

Fig. 2. Two photon microscopy image of FITC fluorescence into DOPC:DPPC:FITCLPS GUVs (1:1:1 molar ratio). The image was taken at $60^{\circ} \mathrm{C}$ where the lipids and LPS are in the liquid-crystalline state. The FITC fluorescence had a homogeneous distribution in GUV's surface.

not favor micelle formation. On the other side DOPC and DPPC have cylindrical shape and as expected they presented lower resistance to TX-100 solubilization at $4{ }^{\circ} \mathrm{C}$.

LPS used in this work (smooth LPS, sLPS) has linked to lipid A a long polysaccharide chain (O-antigen) which also provide an effective steric barrier for inhibiting detergent interaction.

\section{FITC-LPS distribution in GUVs showing macrodomain segregation}

In order to observe the distribution of LPS in the membrane, GUVs of the mixture DOPC:DPPC:FITC-LPS (1:1:1 molar ratio) were observed at two temperatures. Fig. 2 shows the intensity image taken from the top surface of one of these GUVs. The surface appears homogeneously fluorescent. Since the only fluorescent molecule in the chamber is the FITC-LPS (control experiments with unlabeled LPS show no signal) this result indicates that LPS is effectively incorporated into lipid bilayer during the GUVs formation and moreover it shows that, at temperatures at which the two lipids are over their $T_{\mathrm{m}}$, LPS is homogeneously distributed in the bilayer. At $25{ }^{\circ} \mathrm{C}$, however a preferential location of FITC-LPS molecules in some specific areas of the GUV can be observed (Fig. 3A).
A

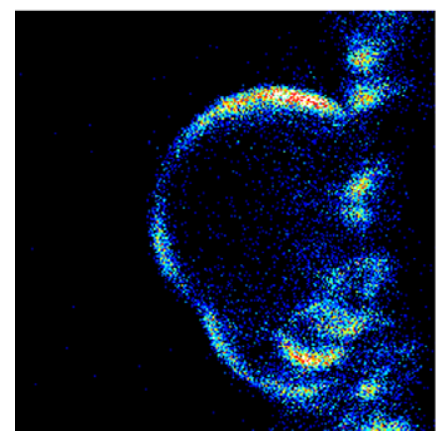

B

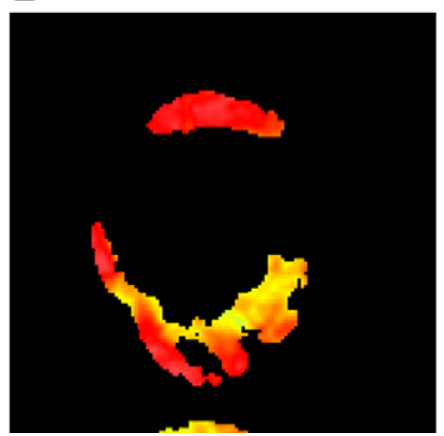

C

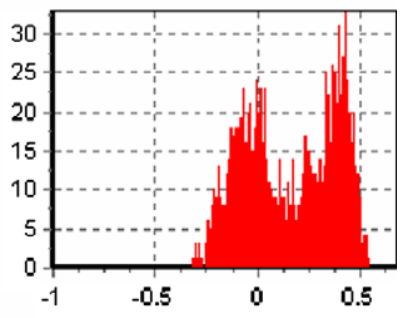

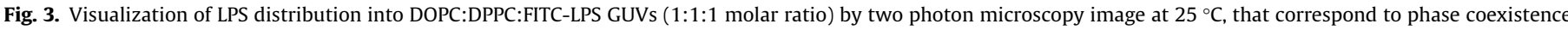

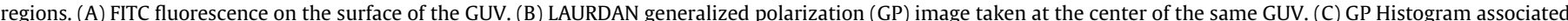

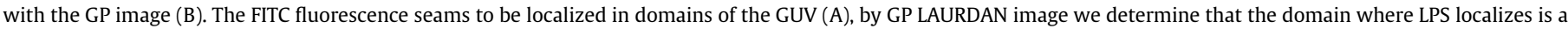
solid domain (B). 
Lipid packing in bilayers containing LPS or cholesterol

To further characterize the nature of the domains in which LPS selectively segregate, LAURDAN was added to the same DOPC:DPPC:FITC-LPS GUV, and the GP image was taken (Fig. 3B). The presence of labeled LPS did not interfere in the GP measurements. Fig. 3B show the GP image of the GUV taken at the equatorial cross section to ensure the excitation of all the LAURDAN molecules with the circular polarized excitation light [11]. A false color scale is used and the image clearly shows areas with different GP values (different colors). The GP histogram (Fig. 3C) corresponds to the distribution of GP values per pixels and show quantitatively what is observed in the image, a bimodal distribution. The fit of the histogram with two distributions gives the average GP of each phase and the corresponding number of pixels, equivalent to the area of any given domain.

The LAURDAN GP image identified the region where FITC-LPS preferentially locate at $25^{\circ} \mathrm{C}$ as the region with high GP (Fig. 3B). These areas are enriched in the lipid that at $25^{\circ} \mathrm{C}$ is below its $T_{\mathrm{m}}$, DPPC.

In order to compare the effect of LPS and cholesterol incorporation into the bilayer, we obtained GP images of the two mixtures (DPPC:DOPC:LPS and DOPC:DPPC:Chol) together with the binary mixtures (DPPC:DOPC) used as a control, at two different temperatures. Fig. 4 shows the comparison of the three mixtures at two temperatures: $55^{\circ} \mathrm{C}$ where all the lipids on the mixture are over their $T_{\mathrm{m}}$, and $25^{\circ} \mathrm{C}$, temperature where DPPC and LPS are below their $T_{\mathrm{m}}$ and DOPC is still over it.

The average GP value is shown in the bar plot and the shape of the domains can be seen in the images taken from the top of the GUVs. At $55^{\circ} \mathrm{C}$, the GP value is homogeneous for the three mixtures and no lipid segregation is observed. The GP value for the mixture containing cholesterol is higher than the control binary mixture, this value is being reported before [6]. However for the mixture containing LPS, the GP value is similar to the binary mixture.

A similar behavior is observed at $25^{\circ} \mathrm{C}$, the GP values for the mixture containing LPS are similar to the control binary mixture and the values in the presence of cholesterol are higher especially for the high GP phase. The cholesterol content for each phase is being determined to be 32 and $22 \mathrm{~mol} \%$ for the lo and ld phase, respectively [25].

Is important to note that the shape of the domains of the mixture containing LPS is similar to the control and it is different from the shape of the domains formed in the sample containing cholesterol.

The LAURDAN GP measurements show that the influence of LPS and cholesterol on the packing of the DOPC:DPPC mixture is different.

LAURDAN GP measurements give information about the packing of the phospholipids in the bilayer based in the rearrangement of few water molecules localized around the LAURDAN dipole located in the naphthalene moiety [26]. Naphthalene moiety locates in the membrane at the level of the phospholipid glycerol backbone and is tightly anchored in the hydrophobic core by the cooperative Van der Waals interactions between the lauric acid tail and the lipid hydrocarbon chains [26]. The planar rings of the cholesterol molecules interact with the hydrocarbon segment of the phospholipids that are closer to the aqueous interface [27] and therefore affect LAURDAN dipole [28]. On the other side, the insertion of LPS in the bilayer is equivalent to the insertion of glycosphingolipids by its lipid A domain. LAURDAN fluorescence in glycosphingolipids show a particular behavior due to the molecular and interfacial structure of this lipids [29]: the bulky polar head confer a more hydrated interface with a higher molecular area than the phospholipids. LAURDAN emission will be affected by both the polarity of the probe's environment and the phase state of the interface.

Under this scenario, one may think that LAURDAN is not reporting accurately the effect of LPS insertion in the bilayer, and the packing of the domains containing LPS and cholesterol are in fact similar. However, the GP images give an extra information about the phases: the shape of the domains. The shape of the domains is related to the packing of the co-existing phases [30] and the fact that the mixture containing LPS and the binary mixture present hand-shaped domains (different from the shape presented in the

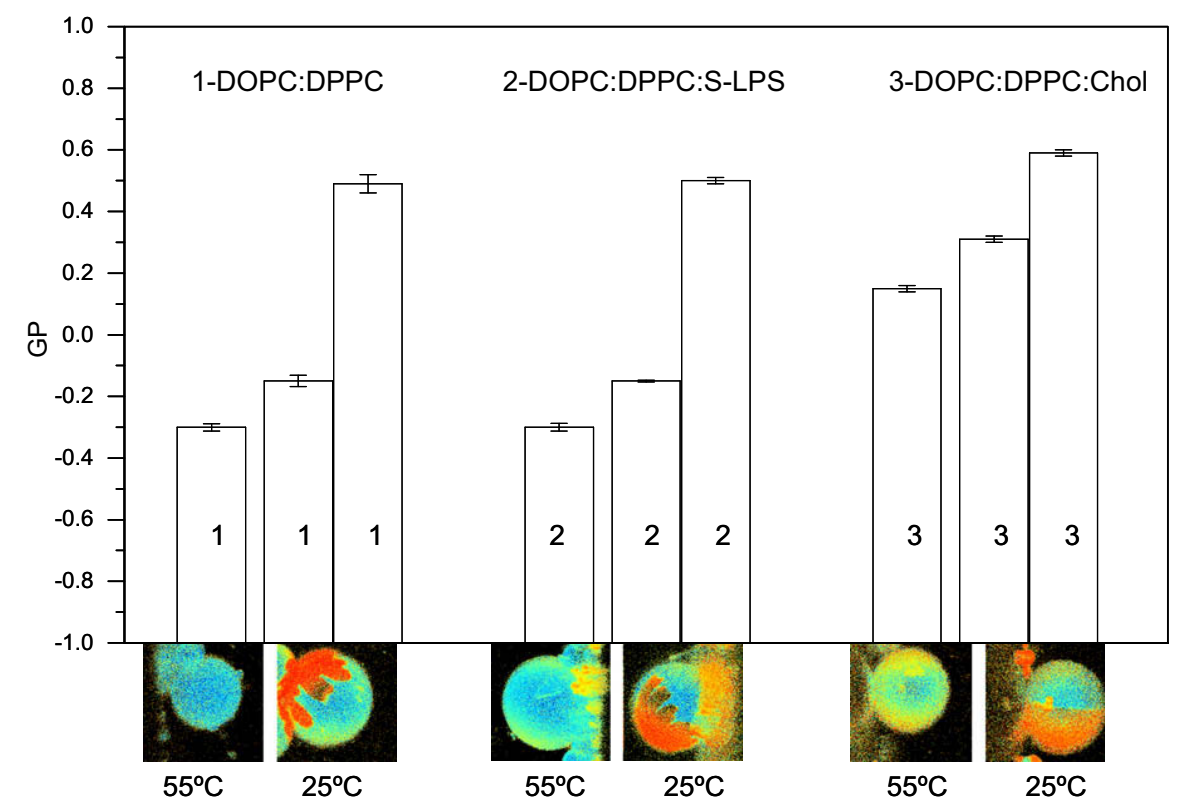

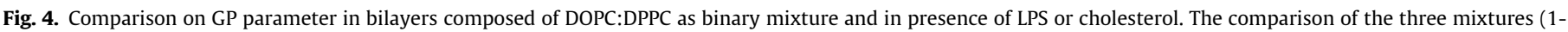

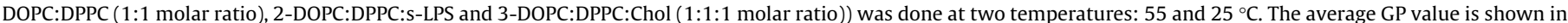

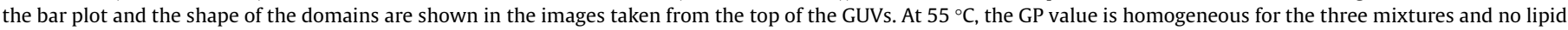
segregation is observed. At $25^{\circ} \mathrm{C}$, where phase segregation occurs, two GP values were obtain, one for each phase. 
mixture containing cholesterol) support the conclusion that the packing of the DPPC domains containing LPS is different from the DPPC domains containing cholesterol. It is important to mention that all the enterobacterial LPS have a gel to liquid-crystalline phase transition in the temperature range $30-37^{\circ} \mathrm{C}$ [31]. At $25^{\circ} \mathrm{C}$ LPS molecules are in their gel state, therefore more prompted to pack together with the DPPC molecules (also bellow their $T_{\mathrm{m}}$ ) than with DOPC molecules in liquid state $\left(T_{\mathrm{m}}\right.$ of $-5{ }^{\circ} \mathrm{C}$ for DOPC and $41^{\circ} \mathrm{C}$ for DPPC) [32].

In this context, we proposed that LPS of $E$. coli could form membrane domains in eukaryotic cell, this domains may be different in nature (different packing) but they can play a similar role than the one play by domains containing cholesterol, organizing a complex orquestration of multiple receptor involved in the signal transduction pathways like it was proposed for $B$. abortus LPS [3]. The presence of LPS domains on eukaryotic cell membranes was described by LPS derived from outer membrane of intracellular pathogens. However, it is possible that LPS delivery from extracellular pathogens in the form of outer membranes vesicles (OMVs) or bacterial membrane fractions could be incorporated into eukaryotic cell membranes through fusion events and then organized by itself in clusters sequestering the components involved in the innate immune response.

\section{Acknowledgments}

This work was supported by Grants from CIC-PBA, ANPCyT (BID 1728/OC-AR 26228), to LB and supported funds for SS from the National Institutes of Health (Grant PHS 5 P41 RR-03155, US).

\section{References}

[1] J. Kriegsmann, S. Gay, R. Brauer, Endocytosis of lipopolysaccharide in mouse macrophages, Cell. Mol. Biol. (Noisy-le-Grand) 39 (1993) 791-800.

[2] C. Forestier, E. Moreno, S. Meresse, et al., Interaction of Brucella abortus lipopolysaccharide with major histocompatibility complex class II molecules in B lymphocytes, Infect. Immun. 67 (1999) 4048-4054.

[3] C. Forestier, F. Deleuil, N. Lapaque, et al., Brucella abortus lipopolysaccharide in murine peritoneal macrophages acts as a down-regulator of T cell activation, J. Immunol. 165 (2000) 5202-5210.

[4] T. Springall, N.S. Sheerin, K. Abe, et al., Epithelial secretion of C3 promotes colonization of the upper urinary tract by Escherichia coli, Nat. Med. 7 (2001) 801-806.

[5] J. Dijkstra, J.W. Mellors, J.L. Ryan, et al., Modulation of the biological activity of bacterial endotoxin by incorporation into liposomes, J. Immunol. 138 (1987) 2663-2670.

[6] S. Sanchez, M.A. Tricerri, E. Gratton, Interaction of high density lipoprotein particles with membranes containing cholesterol, J. Lipid Res. 48 (2007) 16891700.

[7] M.I. Angelova, D.S. Dimitrov, Liposome electroformation, Faraday Discuss. Chem. Soc. 81 (1986) 303-311.

[8] G. Weber, F.J. Farris, Synthesis and spectral properties of a hydrophobic fluorescent probe: 2-dimethylamino-6-propionyl-naphthalene, Biochemistry 18 (1979) 3075-3078.

[9] T. Parasassi, E. Gratton, Membrane lipid domain and dynamics as detected by Laurdan fluorescence, J. Fluoresc. 8 (1995) 365-373.
[10] T. Parasassi, E. Gratton, M. Weiming, et al., Two-photon fluorescence microscopy of Laurdan generalized polarization domains in model and natural membranes, Biophys. J. 72 (1997) 2413-2429.

[11] L. Bagatolli, S. Sanchez, T. Hazlett, et al., Laurdan and two-photon fluorescence microscopy: evidence of lipid lateral separation in bilayers, Methods Enzymol. 360 (2003) 481-500.

[12] M.A. Tricerri, J.D. Toledo, S.A. Sanchez, et al., Visualization and analysis of apolipoprotein A-I interaction with binary phospholipid bilayers, J. Lipid Res. 46 (2005) 669-678.

[13] J.S. Eid, J.D. Muller, E. Gratton, Data acquisition card for fluctuation correlation spectroscopy allowing full access to the detected photon sequence, Rev. Sci. Instrum. 71 (2000) 361-368.

[14] P.T. So, T. French, W.M. Yu, et al., Time-resolved fluorescence microscopy using two-photon excitation, Bioimaging 3 (1995) 49-63.

[15] P.T. So, T. French, W. Yu, et al., Two photon fluorescence microscopy, in: TimeResolved and Intensity Imaging in Fluorescence Imaging Spectroscopy and Microscopy, John Wiley and Sons, New York, 1996.

[16] E. London, D.A. Brown, Insolubility of lipids in Triton X-100: physical origin and relationship to sphingolipid/cholesterol membrane domains (rafts), Biochim. Biophys. Acta 1508 (2000) 182-195.

[17] R.J. Schroeder, E. London, D.A. Brown, Interactions between saturated acyl chains confer detergent resistance on lipids and glycosylphosphatidylinositol (GPI)-anchored proteins: GPI-anchored proteins in liposomes and cells show similar behavior, Proc. Natl. Acad. Sci. USA 91 (1994) 12130-12134.

[18] H. Ahyayauch, B. Larijani, A. Alicia Alonso, et al., Detergent solubilization of phosphatidylcholine bilayers in the fluid state: influence of the acyl chain structure, Biochim. Biophys. Acta 1758 (2006) 190-196.

[19] D. Needham, R.S. Nunn, Elastic deformation and failure of lipid bilayer membranes containing cholesterol, Biophys. J. 58 (1990) 997-1009.

[20] T.J. McIntosh, A.D. Magid, S.A. Simon, Cholesterol modifies the short-range repulsive interactions between phosphatidylcholine membranes, Biochemistry 28 (1989) 17-25.

[21] S. Tristram-Nagle, H.I. Petrache, J.F. Nagle, Structure and interactions of fully hydrated dioleoylphosphatidylcholine bilayers, Biophys. J. 75 (1998) 917-925

[22] S. Snyder, D. Kim, T. McIntosh, Lipopolysaccharide bilayer structure: effect of chemotype, core mutations, divalent cations, and temperature, Biochemistry 38 (1999) 10758-10767.

[23] R.B. Gennis, Biomembranes: Molecular Structure and Function, SpringerVerlag, New York, 1989. p. 533.

[24] A. Schromm, K. Brandenburg, H. Loppnow, et al., Biological activities of lipopolysaccharides are determined by the shape of their lipid A portion, Eur. J. Biochem. 267 (2000) 2008-2013.

[25] S.L. Veatch, S.L. Keller, Separation of liquid phases in giant vesicles of ternary mixtures of phospholipids and cholesterol, Biophys. J. 85 (2003) 3074-3083.

[26] T. Parasassi, E.K. Krasnowska, L.A. Bagatolli, et al., Laurdan and Prodan as polarity-sensitive fluorescent membrane probes, J. Fluoresc. 8 (1998) 365373.

[27] M.B. Sankaram, T.E. Thompson, Cholesterol-induced fluid-phase immiscibility in membranes, Proc. Natl. Acad. Sci. USA 88 (1991) 8686-8690.

[28] T. Parasassi, M. Di Stefano, M. Loiero, et al., Influence of cholesterol on phospholipid bilayers phase domains as detected by Laurdan fluorescence, Biophys. J. 66 (1994) 120-132.

[29] L.A. Bagatolli, B. Maggio, F. Aguilar, et al., Laurdan properties in glycosphingolipid-phospholipid mixtures: a comparative fluorescence and calorimetric study, Biochim. Biophys. Acta 1325 (1997) 80-90.

[30] L.A. Bagatolli, E. Gratton, Two-photon fluorescence microscopy of coexisting lipid domains in giant unilamellar vesicles of binary phospholipid mixtures, Biophys. J. 78 (2000) 290-305.

[31] K. Brandenburg, U. Seydel, Investigation into the fluidity of lipopolysaccharide and free lipid A membrane systems by Fourier-transform infrared spectroscopy and differential scanning calorimetry, Eur. J. Biochem. 191 (1990) 229-236.

[32] R. Koynova, M. Caffrey, Phases and phase transitions of the phosphocholines, Biochim. Biophys. Acta 1376 (1998) 91-145. 